\title{
Enhanced antiproliferative effect of resveratrol in head and neck squamous cell carcinoma using GE11 peptide conjugated liposome
}

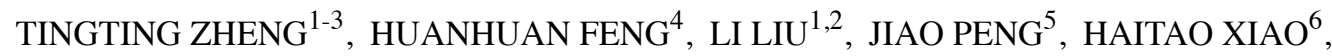 \\ TAO YU ${ }^{7}$, ZIQIAN ZHOU ${ }^{1,2},{\text { YING } \text { LI }^{6} \text {, YUSENG ZHANG }}^{1,2}$, XIAOHE BAI $^{1,2}$, \\ SIMENG ZHAO ${ }^{1,2}$, YU SHI ${ }^{1-3}$ and YUN CHEN ${ }^{1-3}$
}

\begin{abstract}
${ }^{1}$ Shenzhen Key Laboratory for Drug Addiction and Medication Safety, Shenzhen Peking University-Hong Kong University of Science and Technology Medical Center, Shenzhen, Guangdong 510852; ${ }^{2}$ Department of Ultrasound and ${ }^{3}$ Sanming Project of Medicine in Shenzhen, Peking University Shenzhen Hospital, Shenzhen, Guangdong 518036; ${ }^{4}$ Harbin Institute of Technology Shenzhen Graduate School, Shenzhen, Guangdong 510852; ${ }^{5}$ Department of Pharmacy, Peking University Shenzhen Hospital, Shenzhen, Guangdong 518036; ${ }^{6}$ Department of Pharmacy, Health Science Center, Shenzhen University, Shenzhen, Guangdong 518060; ${ }^{7}$ Shenzhen Key Laboratory for Neuronal Structural Biology, Shenzhen Peking University-Hong Kong University of Science and Technology Medical Center, Shenzhen, Guangdong 510852, P.R. China
\end{abstract}

Received August 12, 2018; Accepted January 22, 2019

DOI: 10.3892/ijmm.2019.4096

\begin{abstract}
The present study describes the preparation of a dodecapeptide YHWYGYTPQNVI (GE11)-conjugated liposome bound with polyethylene glycol to enhance the therapeutic effect of resveratrol (RSV) in head and neck cancer cells. The results indicated that (RSV)-loaded GE11-conjugated liposomes (RSV-GL) exhibited a high entrapment efficiency of $>95 \%$, with an active drug loading level of $19.5 \% \mathrm{w} / \mathrm{w}$. Release kinetics revealed that RSV was released in a slow and sustained manner from the RSV-GL and RSV-loaded liposome (RSV-L) nanoparticulate systems. The epidermal growth factor receptor (EGFR)-overexpressing squamous cell carcinoma HN cells specifically internalized GE11 surface-conjugated liposome in a manner that was markedly increased compared with that of the non-targeted carrier. Consistently, RSV-GL exhibited a significantly increased cytotoxic effect compared with that of the non-targeted nanoparticles. Notably, RSV-GL induced significantly increased proportions of early $(\sim 60 \%)$ and late $(\sim 10 \%)$ apoptotic cells in head and neck cancer cell populations. To the best of our knowledge, the application and development of EGFR-targeted peptide-conjugated liposome system for RSV delivery has not been studied previously in the treatment of head and neck cancer. In addition, RSV-GL
\end{abstract}

Correspondence to: Dr Yun Chen, Shenzhen Key Laboratory for Drug Addiction and Medication Safety, Shenzhen Peking University-Hong Kong University of Science and Technology Medical Center, 1120 Lianhua Road, Shenzhen, Guangdong 510852, P.R. China

E-mail: cdevriesott@yahoo.com

Key words: resveratrol, head and neck cancer, apoptosis, liposome, epidermal growth factor receptors exhibited the greatest antitumor efficacy compared with any other group. RSV-GL exhibited a 2-fold decrease in tumor volume compared with the free RSV and a 3-fold decrease in volume compared with the control. Overall, the nanomedicine strategy described in the present study may potentially advance the chemotherapy-based treatment of head and neck cancer, with promising applications in other EGFR-overexpressing tumors.

\section{Introduction}

Head and neck squamous cell carcinoma (SCC) constitutes an entire group of epithelial cancer types, including cancer of the lip, oral, salivary glands, pharynx and larynx (1). According to one estimate, 600,000 incident cases of HNSCC occur every year worldwide (2). At present, surgery is performed in combination with radiotherapy or chemotherapy to treat cancer of the head and neck $(3,4)$. However, these treatment strategies generally result in significant side effects and decreases in patient quality of life. In particular, chemotherapy started at an early stage may be effective; however, cancer cells often develop resistance and delivering large amounts of the drug locally to specific tumor sites remains a challenge $(5,6)$. Therefore, the development of strategies that increase the local concentration of drugs in the tumor tissues is required.

In this regard, resveratrol (RSV), a naturally occurring polyphenolic compound (flavonoid) has been demonstrated to exhibit multiple key properties, including anti-inflammatory, anti-oxidant, anti-aging, cardio-neuro protective and antitumor effects (7-9). RSV serves an important role in the suppression of cancer cell proliferation, tumor ablation, angiogenesis and cancer metastasis (10). RSV has been revealed to inhibit the proliferation of HL60, MCF-7, SW480 and U251 glioma cells by inducing apoptosis $(11,12)$. However, the efficacy of RSV is limited due to its low levels of solubility; therefore, the present 
study aimed to combine RSV with a nanocarrier and evaluate its anticancer effect in head and neck cancer (13).

Nanoparticles have been wide investigated as drug delivery carriers with cancer targeting applications. A poorly soluble drug may be stably incorporated in the nanoparticles and thereby its solubility and bioavailability is improved (14-16). Among all the carriers, liposomes have been widely studied for their suitability for systemic application (17). At present, a number of commercial liposomal formulations are available including Doxil ${ }^{\circledR}$, Myocet ${ }^{\circledR}$ and LipoPlatin $(18,19)$. The systemic circulation of liposomes may be improved by the surface conjugation of polyethylene glycol (PEG) as a hydrophilic shell. The nanosize and hydrophilic layer of PEG confers prolonged blood circulation and decreases the level of reticuloendothelial (RES)-mediated plasma clearance $(20,21)$. Nanoparticles may passively accumulate in the tumor tissues via the enhanced permeation and retention (EPR) effect. In order to additionally increase the cancer specificity, liposomes may be conjugated with specific ligands that bind to the extracellular domains of cancer cells (22). Among all the molecular targets, epidermal growth factor receptor (EGFR) is overexpressed in head and neck cancer and its activation is expected to inhibit the tumor cell proliferation and enhance apoptosis in cancer cells $(23,24)$. Various EGFR inhibitors include tyrosine kinase inhibitors, immunoconjugates, oligonucleotides and epidermal growth factor (EGF). A number of studies have conjugated EGF on the surface of nanoparticles to target cancer cells (25-27). In the present study, the dodecapeptide YHWYGYTPQNVI (GE11) was conjugated onto the surface of nanoparticles. The GE11 peptide is able to selectively bind to the EGFR but with decreased mitogenic activity compared with that of other EGF blockers (28).

In the present study, liposomes and nanoparticles conjugated with the GE11 surface peptide were prepared to increase the anticancer effects of RSV. We hypothesized that surface conjugation of the liposome with the targeting ligand would improve the anticancer effect effectively compared with that of non-targeted nanoparticles. The targeting efficiency of the nanoparticles was examined in squamous cell carcinoma (SCC) HN cancer cells. The anticancer effect was evaluated using MTT cytotoxicity and apoptosis assays in EGFR-overexpressing SCC HN cells.

\section{Materials and methods}

Materials. Egg L-a-phosphatidylcholine (EPC) and disteroylphosphatidylethanolamine-PEG (2000) (DSPE-PEG), and 1,2-distearoyl-sn-glycero-3-phosphoethanolamine-NPEG-maleimide (DSPE-PEG2000-MAL) were purchased from Avanti PolarLipids, Inc. (Alabaster, AL,USA). Cholesterol and RSV were purchased from Sigma-Aldrich; Merck KGaA (Darmstadt, Germany). GE11 (YHWYGYTPQNVIGGGGC), which is a specific peptide for EGFR, was purchased from GL Biochem (Shanghai) Ltd. (Shanghai, China). All other chemicals were of reagent grade and used without additional purification.

Preparation of RSV-loaded GE11-conjugated liposomes. The RSV-loaded liposome (RSV-L) was prepared by a thin-film hydration technique. In brief, EPC, DSPE-PEG, DSPE-PEG-Mal and cholesterol at a molar fraction of 59:10:5:26 were added to $2 \mathrm{ml}$ chloroform and placed in a round bottomed flask. Then, $20 \%$ w/w RSV was also added (20\% w/w lipid). The organic solvents were evaporated by rotary evaporator by rotation $(0.2 \mathrm{xg})$ at $60^{\circ} \mathrm{C}$ for $1 \mathrm{~h}$ and to produce a thin film. PBS was added to the thin film and hydrated for $1 \mathrm{~h}$ at $60^{\circ} \mathrm{C}$. The suspension was then extruded 21 times through a polycarbonate membrane with a pore size of $200 \mathrm{~nm}$ (Avanti Polar Lipids, Inc.). The drug-loaded liposomes were stored in a refrigerator until use. To conjugate the peptide, GE11 was dissolved in HEPES buffer (Sigma-Aldrich; Merck KGaA) and reacted with liposomes containing DSPE-PEG-Mal (1:5 molar ratio) and incubated at $24^{\circ} \mathrm{C}$ for $15 \mathrm{~h}$ in the dark. The unconjugated GE11 was removed by ultracentrifugation at $24^{\circ} \mathrm{C}$ and $4,000 \mathrm{x} \mathrm{g}$ for $15 \mathrm{~min}$.

Particle size and morphology. The average particle size and polydispersity index, a measure of uniformity of particle size distribution and surface charge, were evaluated by dynamic light scattering using a Nano-Z590 Zetasizer instrument (Malvern Instruments, Ltd., Malvern, UK). The suspension was diluted with distilled water and examined at $25^{\circ} \mathrm{C}$. The experiment was performed in triplicate using 3 independent samples. The morphology of the particles were evaluated using transmission electron microscopy (TEM) by field-emission TEM using a JEM-2100F microscope (JEOL, Ltd., Tokyo, Japan). The suspension was stained with $2 \%$ phosphotungstic acid and placed on a copper grid for $15 \mathrm{~min}$ at $24^{\circ} \mathrm{C}$. The sample was dried and observed under a microscope at x10,000.

In vitro release study. The dialysis bag was immersed in water for $8 \mathrm{~h}$ prior to the commencement of the study. The samples (RSV-L and RSV-GL; $1 \mathrm{ml}$ drug-loaded suspension) were packed in the dialysis bag and ends were sealed appropriately. The bag was immersed in PBS ( $\mathrm{pH} \mathrm{7.4)} \mathrm{and} \mathrm{maintained} \mathrm{at}$ $37^{\circ} \mathrm{C}$. The samples were collected at pre-determined time intervals and replaced with equal amounts of fresh buffer. The amount of drug released in the medium was calculated using a high performance liquid chromatography (HPLC) method. A $10 \mu \mathrm{l}$ sample was used. A Jasco HPLC system (pump PU-2089, autosampler AS-2057 and LC-Net II/ADC controller; Jasco, Inc., Easton, MD, USA) was coupled to a fluorometric detector (Jasco FP-2020, $\lambda$ excitation $=330 \mathrm{~nm}$ and $\lambda$ emission $=374 \mathrm{~nm})$. Synergi $4 \mu$ HydroRP 80A and Luna $5 \mu$ 100A C18 analytical columns $(250 \times 4.60 \mathrm{~mm}$; Phenomenex, Inc., Torrance, CA, USA) were used. The mobile phase comprised of methanol: ACN: $0.1 \%$ phosphoric acid in water $(60: 10: 30 \mathrm{v} / \mathrm{v})$ with a flow rate of $1 \mathrm{ml} / \mathrm{min}$. Ibuprofen was used as the internal standard, and injection volume was $20 \mu \mathrm{l}$ with flow rate of $1 \mathrm{ml} / \mathrm{min}$ and isocratic flow was adapted.

Cell culture. The SCC HN cancer cell line (SCC-VII) was purchased from Soochow University Cell Bank (Suzhou, China) and grown in Dulbecco's modified Eagle's medium (Thermo Fisher Scientific, Inc., Waltham, MA, USA) media supplemented with $10 \%$ fetal bovine serum (Thermo Fisher Scientific, Inc.) and $1 \%$ antibiotic mixture. The cells were grown at $37^{\circ} \mathrm{C}$ with $65 \%$ humidity.

Cellular uptake of GE11-conjugated liposomes. Rhodamine-B (Sigma-Aldrich; Merck KGaA) was used as a fluorescent probe 
to track the intracellular uptake of nanoparticles. The SCC HN cells were seeded in a 6 -well plate $\left(3 \times 10^{5}\right)$ and incubated overnight at $37^{\circ} \mathrm{C}$. The cells were then exposed to $10 \mu \mathrm{g} \mathrm{RSV}-\mathrm{L}$ and RSV-loaded GE11-conjugated liposomes (RSV-GL) and incubated for $2 \mathrm{~h}$ at $37^{\circ} \mathrm{C}$. The cells were then washed and extracted using 5\% trypsin. The cells were washed again and reconstituted in a PBS buffer. The cellular internalization of the RSV-L and RSV-GL was studied using a FACSCalibur flow cytometer (BD Biosciences, San Jose, CA, USA). BD CellQuest Pro software (version 5.1; BD Biosciences).

Cytotoxicity assay. The cytotoxicity potential of individual formulation was evaluated by a CellTiter $96^{\circledR}$ AQueous One Solution Cell Proliferation MTT assay (Promega Corporation, Madison, WI, USA). The cells were seeded at a density of $1.5 \times 10^{4}$ cells/well in a 96-well plate and incubated for $24 \mathrm{~h}$ at $37^{\circ} \mathrm{C}$. The old medium was replaced with new medium containing unbound RSV, RSV-L and RSV-GL, and incubated at $37^{\circ} \mathrm{C}$ for $24 \mathrm{~h}$ with $50 \mu \mathrm{g}$ liposome in each group. The following day, the medium was removed, and cells were washed twice with PBS. The cells were treated with MTS solution as per the manufacturer's protocol. The absorbance of each well was studied using a microplate reader a $490 \mathrm{~nm}$. Control cells were treated with dimethyl sulfoxide alone and a separate control was established using non-treated cells. All experiments were performed in triplicate.

Apoptosis assay. The apoptosis of cancer cell was studied using an Annexin V-fluorescein isothiocyanate (FITC)/propidium iodide (PI) staining protocol. Briefly, cells were seeded at a density of $2 \times 10^{5}$ cells/well in a 12 -well plate and incubated for $24 \mathrm{~h}$ at $37^{\circ} \mathrm{C}$. The old medium was replaced with new medium containing unbound RSV, RSV-L and RSV-GL $(0.1$ to $10 \mu \mathrm{g} / \mathrm{ml})$, respectively and incubated for $24 \mathrm{~h}$ at $37^{\circ} \mathrm{C}$. Following incubation, cells were washed twice with PBS and extracted. The cells were centrifuged at $200 \mathrm{x} \mathrm{g}$ for $4 \mathrm{~min}$ at $8^{\circ} \mathrm{C}$, and the pellets was reconstituted with binding buffer (100 $\mu \mathrm{l}$; BD Biosciences). The cells were stained with $2 \mu \mathrm{l}$ Annexin V-FITC and $2 \mu \mathrm{l}$ PI (BD Biosciences) and incubated for $15 \mathrm{~min}$ at $24^{\circ} \mathrm{C}$. The volume was made up to $1 \mathrm{ml}$ and examined using a FACSCalibur flow cytometer (BD Biosciences) and CellQuest Pro software (version 5.1; BD Biosciences).

In vivo antitumor efficacy analysis and immunostaining. The animal study was approved by the Institutional Animal Ethical Committee of the Shenzhen Peking University-Hong Kong University of Science and Technology Medical Center (Shenzhen, China). Female nude mice ( 20 g; 5 weeks old) were purchased from the Chinese Academy of Sciences (Shanghai, China). Animals were housed in separate cages (16 animals with 4 animals per cage) and maintained under a controlled atmosphere $\left(50 \pm 7 \%\right.$ humidity, $\left.21 \pm 1^{\circ} \mathrm{C}\right)$ with a $12 \mathrm{~h}$ light/dark cycle. The animals had free access to food and water throughout the study period. The tumor xenograft model was developed by injecting $1 \times 10^{6}$ cells in $100 \mu 1$ growth media into the right flank of female $\mathrm{BALB} / \mathrm{c}$ nude mice. The tumors were allowed to grow until $100 \mathrm{~mm}^{3}$ and then experiments were initiated. The mice were randomly divided into four groups: The control; RSV; RSV-L; and RSV-GL groups, with 8 mice in each group. The mice were injected with $10 \mathrm{mg} / \mathrm{kg} \mathrm{RSV}$ (equivalent concentration) three times (with 3-day intervals) via a tail vein injection. The tumor volume was measured using Vernier Caliper and calculated using the formula $\mathrm{V}=\left(\right.$ length $\mathrm{x}$ width $\left.{ }^{2}\right) / 2$. All animals were sacrificed, and tumors were surgically removed and examined histologically. Hematoxylin and eosin (H\&E) staining was performed on the tumor slices following fixing with $10 \%$ formalin solution for $20 \mathrm{~min}$ at $24^{\circ} \mathrm{C}$. The images were obtained at $\mathrm{x} 40$ magnification using a fluorescence microscope (1X81; Olympus Corporation, Tokyo, Japan).

Statistical analysis. Statistical analysis was performed using Student's t-test for pairs of groups and one-way analysis of variance followed by Tukey's post hoc test for multiple groups in Excel (Microsoft Corporation, Redmond, CA, USA). All data are expressed as the mean \pm standard deviation. $\mathrm{P}<0.05$ was considered to indicate a statistically significant difference. For the cell viability assay, data were assessed using GraphPad Prism software v.7.0 (GraphPad Software, Inc., La Jolla, CA, USA).

\section{Results and Discussion}

In the present study, the dodecapeptide GE11 was conjugated onto the surface of nanoparticles (Fig. 1). RSV, which serves an important role in the suppression of cancer cell proliferation, tumor ablation, angiogenesis and cancer metastasis, was selected as the nanoparticle. We hypothesized that surface conjugation of liposome with targeting ligand would improve the anticancer effect effectively compared with that of non-targeted nanoparticles.

Physicochemical characterizations of RSV-GL. The average particle size of RSV-GL was observed to be $\sim 185 \mathrm{~nm}$ compared with $\sim 130 \mathrm{~nm}$ for RSV-L (Table I) with a surface charge of $-23.4 \pm 0.98 \mathrm{mV}$. The increase in particle size in the case of RSV-GL was primarily attributed to the conjugation of GL on its surface (Fig. 2). The particle size of RSV-GL in culture medium was observed to be $\sim 208 \mathrm{~nm}$. A slight increase in the particle size in culture medium may be due to the adsorption of proteins on the surface. It has been suggested that nanocarriers with an average size between 100-200 $\mathrm{nm}$ will extravasate into the tumor tissues preferentially via the EPR effect (25). General features of tumors include leaky blood vessels and poor lymphatic drainage. In these conditions, a nanocarrier may extravasate into the tumor tissues via the leaky vessels by the EPR effect. The dysfunctional lymphatic drainage in tumors retains the accumulated nanocarriers and allows them to release drugs into the vicinity of the tumor cells. Experiments using liposomes of different mean sizes have suggested that the threshold vesicle size for extravasation into tumors is $\sim 400 \mathrm{~nm}(29,30)$, but previous studies have indicated that particles with diameters $<200 \mathrm{~nm}$ are more effective (20). In addition, the presence of PEG on the outer surface will increase the blood circulation. The small particle size also allows it to escape from RES-based systemic clearances (31).

Entrapment efficiency and loading efficiency are two important parameters that determine the entrapment capacity of the liposomal carrier. The results of the present study demonstrated that RSV-GL exhibited a high entrapment 
Table I. Physicochemical characteristics of drug-loaded formulations.

\begin{tabular}{lccccc}
\hline Nanoparticles & Size, $\mathrm{nm}$ & Surface charge, $\mathrm{mV}$ & Polydispersity index & Entrapment efficiency, \% & Loading efficiency, $\%$ \\
\hline Blank L & $104.3 \pm 1.65$ & $-21.4 \pm 1.14$ & 0.089 & - & - \\
RSV-L & $129.5 \pm 1.65$ & $-24.1 \pm 1.19$ & 0.112 & $96.2 \pm 1.18$ & $8.95 \pm 1.28$ \\
RSV-GL & $187.5 \pm 2.13$ & $-23.4 \pm 0.98$ & 0.145 & $94.2 \pm 1.26$ & $7.45 \pm 1.57$ \\
\hline
\end{tabular}

RSV, resveratrol; L, GL, GE11-conjugated liposomes.

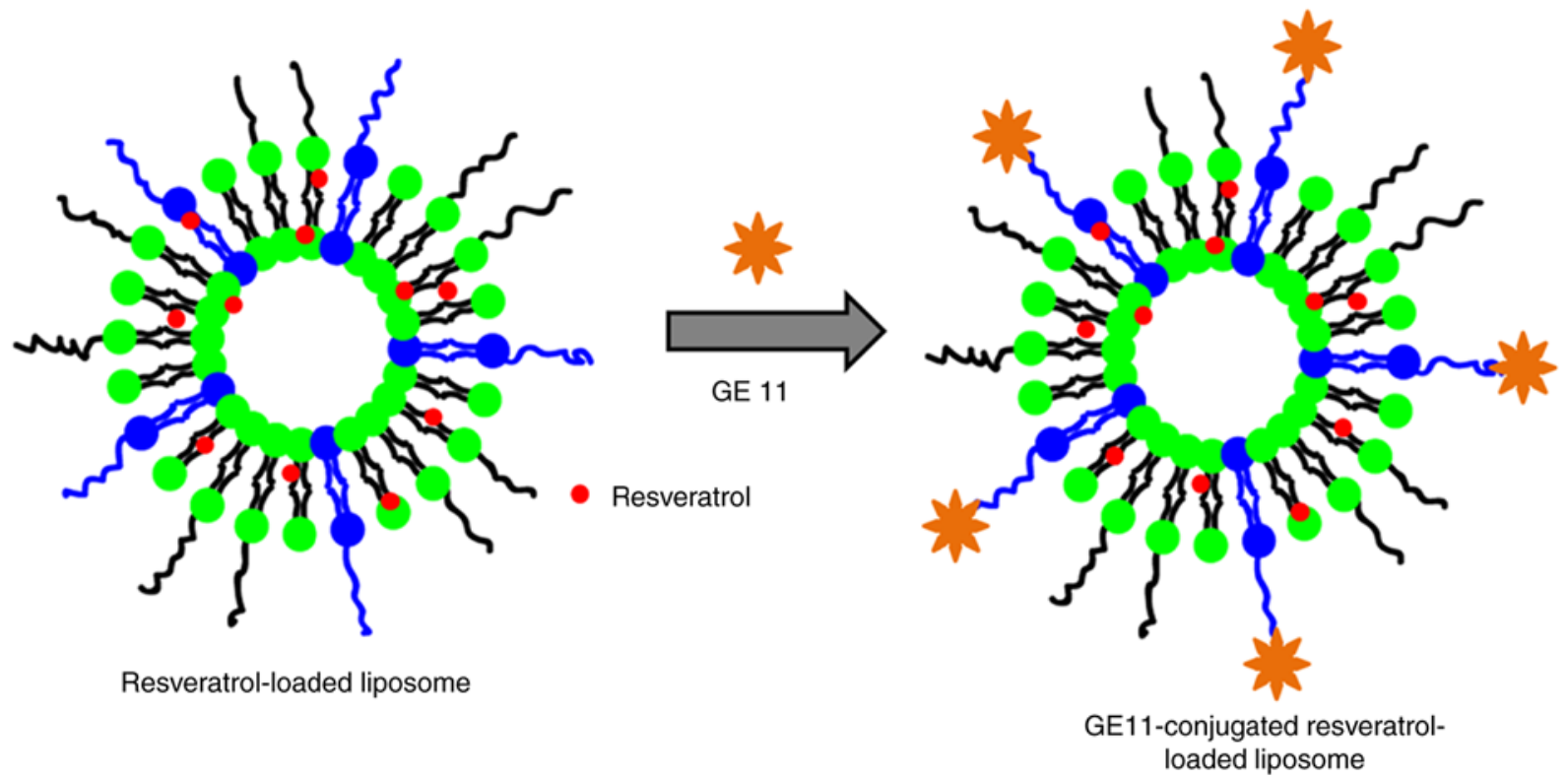

Figure 1. Schematic illustration of preparation of GE11-conjugated resveratrol-loaded liposomal formulations. GE11, dodecapeptide YHWYGYTPQNVI.

efficiency of $>95 \%$ with an active drug loading $19.5 \% \mathrm{w} / \mathrm{w}$, indicating the excellent characteristics of the present carrier (Table I). This may be attributed to the fact that RSV has high hydrophobic characteristics that allow integration into the lipid bilayer of the liposome.

In vitro drug release study. The drug release study was performed in PBS (pH 7.4) at $37^{\circ} \mathrm{C}$. It was observed that RSV was released in a slow and sustained manner from the RSV-L and RSV-GL nanoparticulate systems (Fig. 3). For example, $\sim 30 \%$ of RSV was released from the nanocarriers after $24 \mathrm{~h}$, while $\sim 70 \%$ of the drug was released after $72 \mathrm{~h}$ from RSV-GL. The slightly decreased drug release rate observed in the RSV-GL group compared with RSV-L was primarily attributed to the presence of GL on the outer surface. During initial time points, significant differences between the RSV-L and RSV-GL nanoparticles were observed $(\mathrm{P}<0.05)$. It should be noted that no initial high rate of release was observed, indicating that all of the drugs were stably loaded into the cores of the nanoparticles, and none had been absorbed onto the surface of the carrier. This is crucial, as a sustained release of drugs from the nanocarrier system will be a beneficial characteristic for its proposed cancer-targeting applications (32).

In vitro cellular uptake analysis. The cellular uptake efficiency of individual nanoparticles was evaluated by FACS analysis. The cancer cells were treated with respective formulations and cellular uptake was observed after $2 \mathrm{~h}$ incubation (Fig. 4). As observed, rhodamine-B loaded RSV-L exhibited definite internalization of the carrier in the cancer cells, while RSV-GL exhibited significantly increased cellular uptake in cancer cells compared with the RSV-L nanoparticles. The EGFR-overexpressing SCC HN cells specifically internalized the GL11 surface conjugated liposome in a manner that was markedly increased compared with that of the non-targeted carrier. The liposomes were internalized via a specific receptor-mediated active internalization triggered by the binding of the GE11 peptides to the EGFR-overexpressing head and neck cancer cells. This increased cellular uptake of nanocarrier is expected to increase the therapeutic efficacy in cancer cells $(33,34)$.

In vitro cytotoxicity assay. The in vitro cytotoxicity of individual formulations was evaluated by MTT assay. As observed, unbound RSV and RSV-loaded nanoparticles exhibited a typical time-dependent cytotoxic effect in head and neck cancer cells (Fig. 5). It was observed that NP encapsulation of RSV increased the therapeutic effect in cancer cells. To be specific, RSV-GL exhibited a significantly increased cytotoxic effect compared with that of the non-targeted nanoparticles $(\mathrm{P}<0.05)$. The half maximal inhibitory concentration values of unbound RSV, RSV-L and RSV-GL were 4.5, 22.5 and 

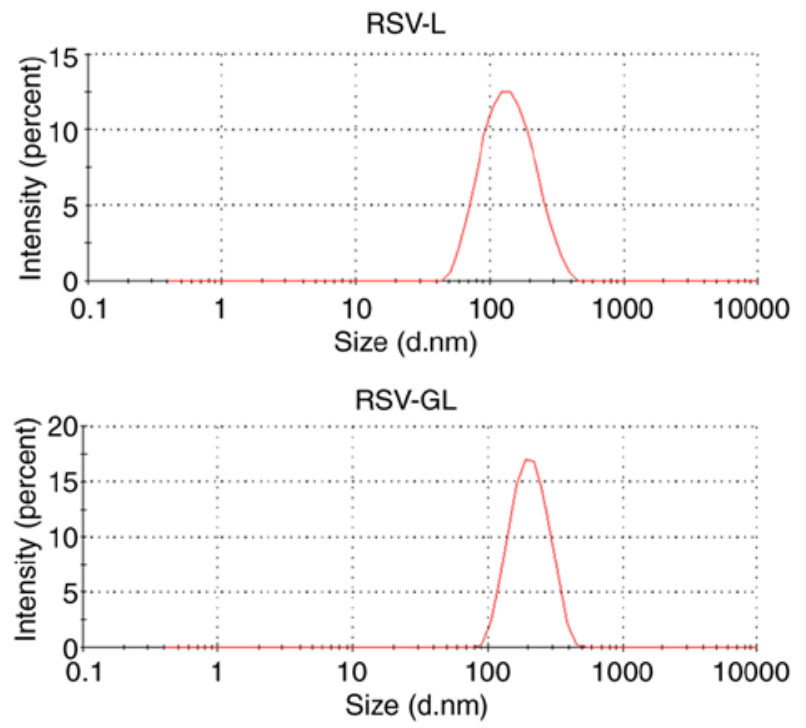

Figure 2. Particle size distribution of RSV-L and RSV-GL. The particle size evaluated by means of dynamic light scattering analysis. RSV, resveratrol; GE11, dodecapeptide YHWYGYTPQNVI; RSV-L, RSV-loaded liposome; RSV-GL, RSV-loaded GE11-conjugated liposomes.

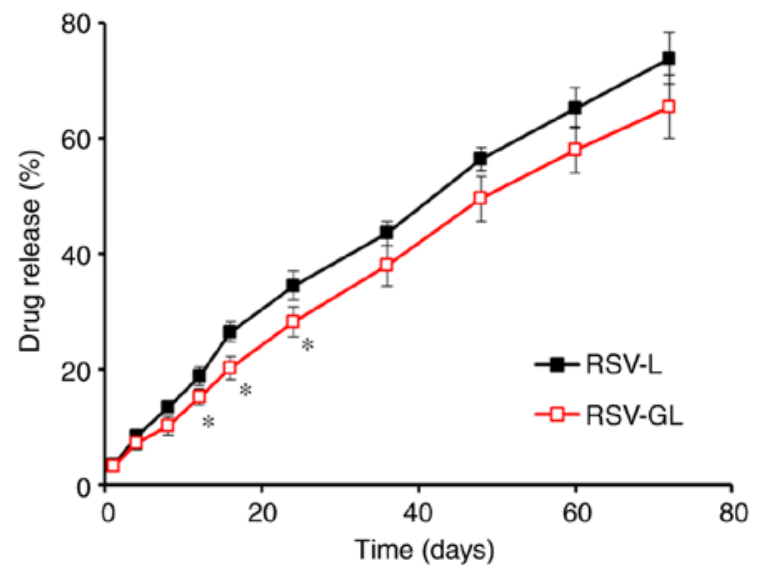

Figure 3. In vitro release study of RSV from RSV-L and RSV-GL. The release of the drug from the nanoparticle was evaluated by means of high-performance liquid chromatography. ${ }^{*} \mathrm{P}<0.05$ vs. RSV-L. RSV, resveratrol; GE11, dodecapeptide YHWYGYTPQNVI; RSV-L, RSV-loaded liposome; RSV-GL, RSV-loaded GE11-conjugated liposomes.

$34.6 \mu \mathrm{g} / \mathrm{ml}$, respectively. The superior anticancer effect of RSV-GL was attributed to the specific receptor-mediated active internalization of RSV-GL, which triggered the binding of the GE11 peptides to the EGFR-overexpressing head and neck cancer cells and resulted in increased intracellular concentrations and a cytotoxic effect.

Apoptosis assay. The antitumor efficacy of individual formulations was additionally evaluated by an apoptosis assay using a flow cytometer (Fig. 6). For this assay, cells were treated with respective formulations and then stained with an Annexin V/PI staining kit. A total of $\sim 9 \%$ of the cells treated with RSV were identified to be in early apoptosis. Conversely, RSV-L induced a relatively increased apoptosis effect, with $\sim 17.5 \%$ in early and $\sim 4 \%$ in late apoptosis. Notably, RSV-GL induced a significantly increased early $(\sim 60 \%)$ and late $(\sim 5 \%)$ apoptotic

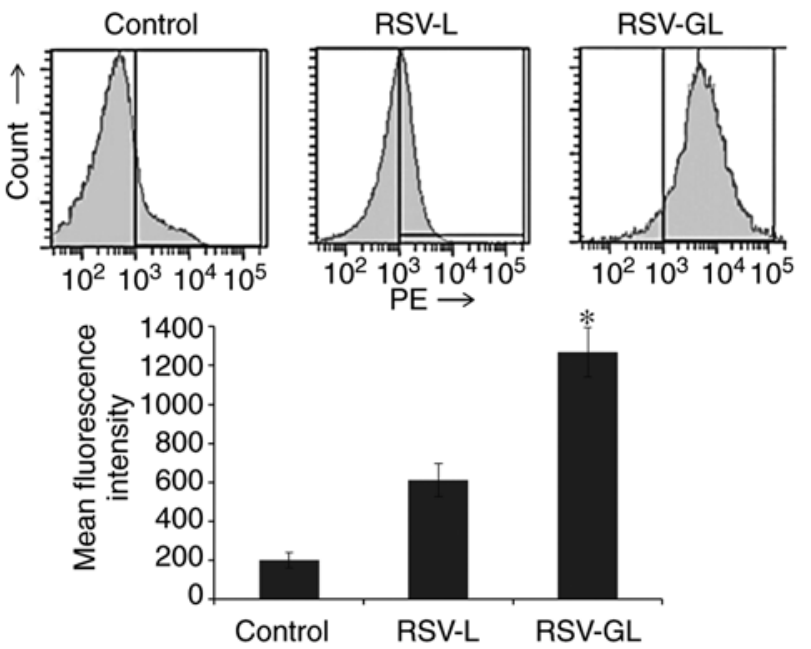

Figure 4. In vitro cellular uptake analysis of RSV-L and RSV-GL using FACS flow cytometry. The rhodamine-B was used as a fluorescent probe to evaluate cellular uptake. "P<0.05 vs. RSV-L. RSV, resveratrol; GE11, dodecapeptide YHWYGYTPQNVI; RSV-L, RSV-loaded liposome; RSV-GL, RSV-loaded GE11-conjugated liposomes.

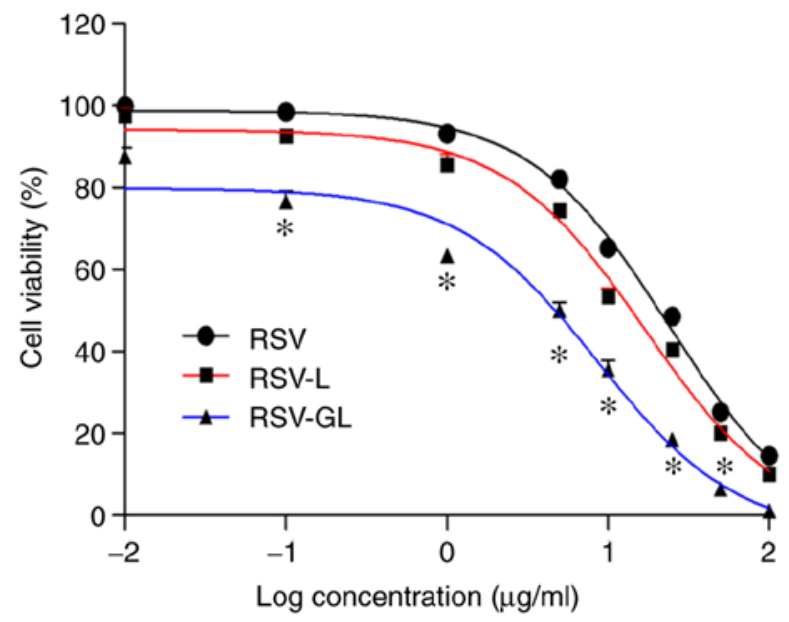

Figure 5. In vitro cytotoxicity assay of unbound RSV, RSV-L and RSV-GL in head and neck cancer cells. The cytotoxicity assay was determined by MTT assay. "P<0.05 vs. RSV-L. RSV, resveratrol; GE11, dodecapeptide YHWYGYTPQNVI; RSV-L, RSV-loaded liposome; RSV-GL, RSV-loaded GE11-conjugated liposomes.

effect in head and neck cancer cells $(\mathrm{P}<0.01)$ compared with the RSV-L-treated cells. The increased populations of early and late apoptotic cells in the RSV-GL-treated cancer cells compared with control demonstrates an enhanced anticancer effect of the ESV-GL nanoparticle system. The increased proportion of cells in early apoptosis compared with late apoptosis may be due to the limited incubation period of $24 \mathrm{~h}$. The enhanced apoptosis effect was due to the enhanced cellular accumulation of nanoparticles attributed to the receptor-mediated endocytosis (35).

In vivo antitumor efficacy and immunohistology analysis. The in vivo antitumor efficacy was performed in SCC-bearing xenograft mice (Fig. 7A). As demonstrated, tumors in the untreated animal model grew continuously at every time point. The unbound RSV exhibited a limited effect on tumor growth, 


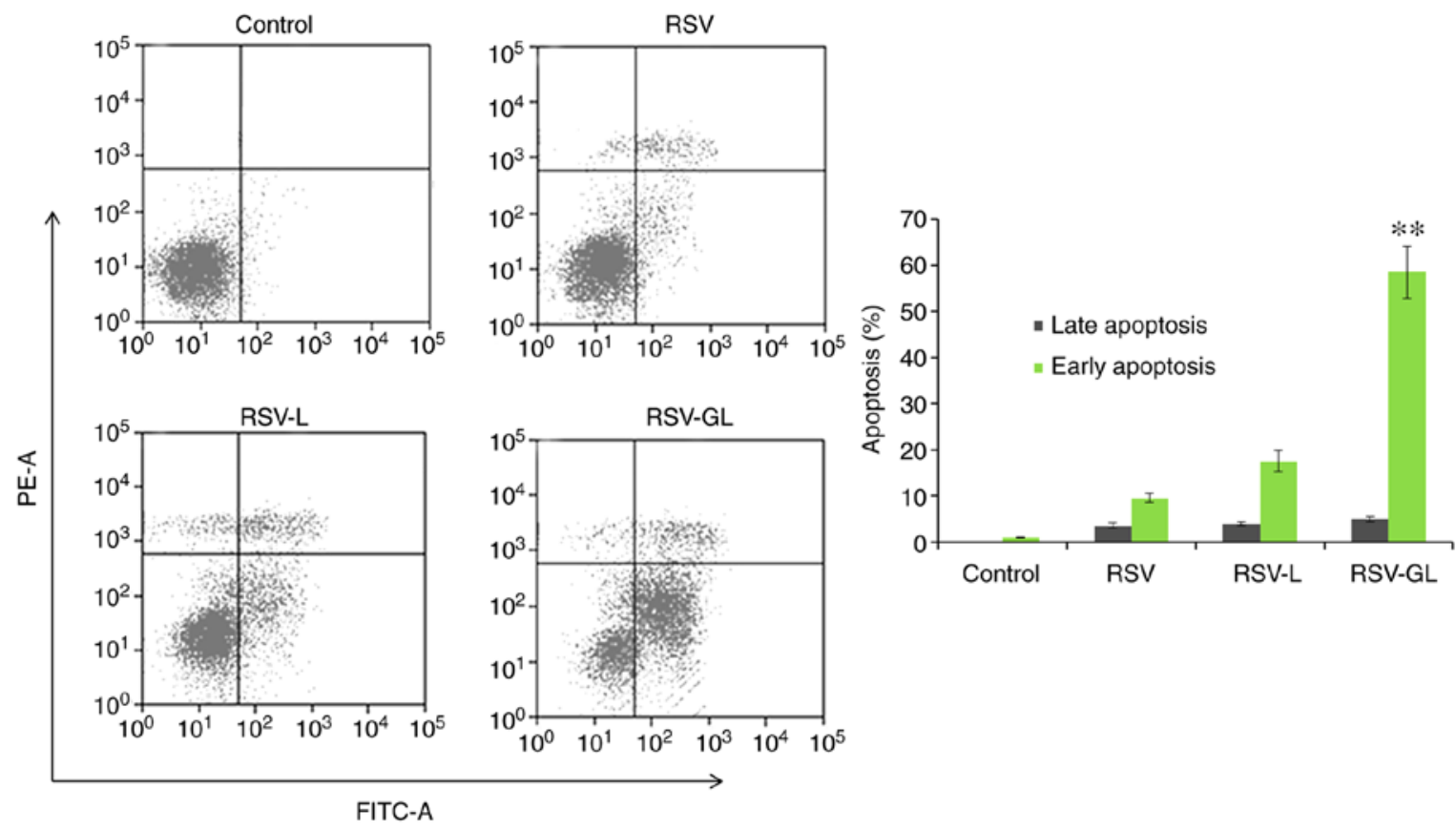

Figure 6. Apoptosis analysis of unbound RSV, RSV-L and RSV-GL in head and neck cancer cells. The apoptosis of cancer cells was studied by Annexin V-FITC/PI staining. ${ }^{* *}$ P $<0.01$ vs. RSV-L. RSV, resveratrol; GE11, dodecapeptide YHWYGYTPQNVI; RSV-L, RSV-loaded liposome; RSV-GL, RSV-loaded GE11-conjugated liposomes; FITC, fluorescein isothiocyanate; PI, propidium iodide.

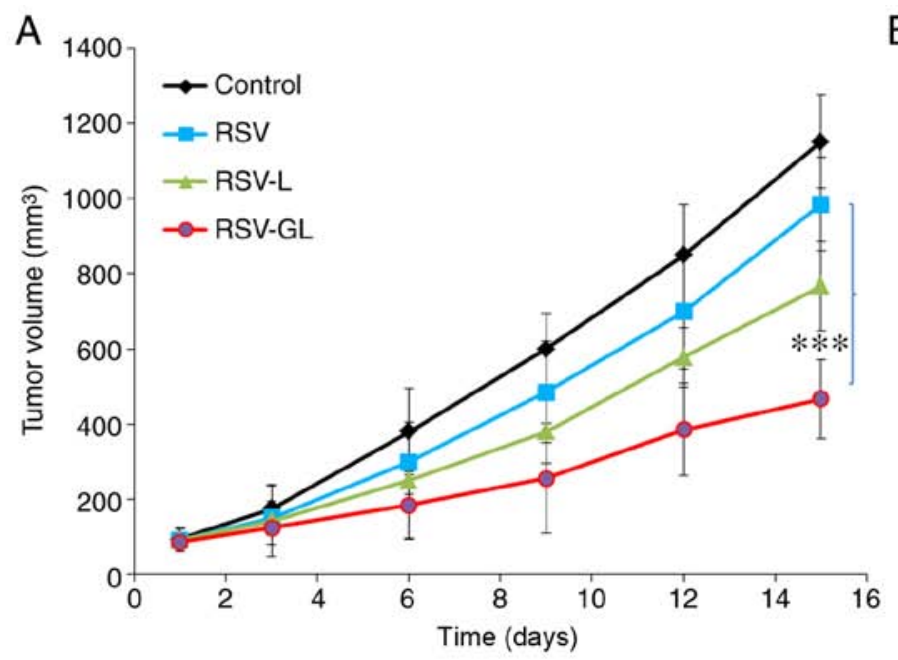

B
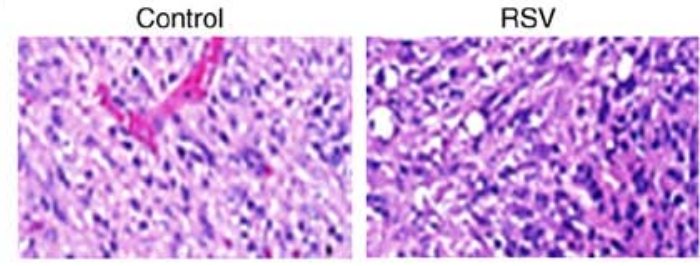

RSV-L

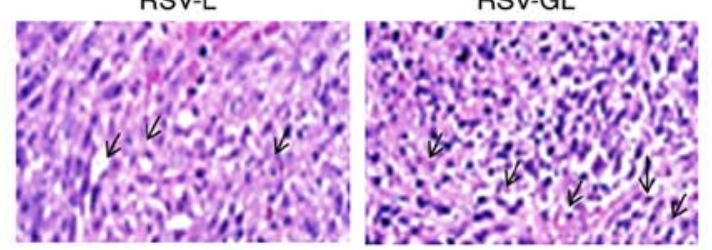

Figure 7. In vivo anticancer efficacy study in squamous cell carcinoma-bearing xenograft model. (A) Tumor volume measurement. (B) Hematoxylin and eosin histology staining analysis. The animals were treated with respective formulations (unbound RSV, RSV-L and RSV-GL) and the experiments were initiated when the tumor volumes reached $\sim 100 \mathrm{~mm}^{3}$. $^{* * *} \mathrm{P}<0.0001$ vs. RSV. RSV, resveratrol; GE11, dodecapeptide YHWYGYTPQNVI; RSV-L, RSV-loaded liposome; GL, GE11-conjugated liposomes.

and the effect on tumor growth of RSV-L nanoparticle was slightly improved compared with the unbound RSV group. Notably, RSV-GL demonstrated significant antitumor efficacy compared with any other group $(\mathrm{P}<0.0001)$. RSV-GL exhibited a 2-fold decrease in tumor volume compared with the free RSV group, and a 3-fold decrease in volume compared with the control. The final tumor volumes were 1,152, 985, 768 and $467 \mathrm{~mm}^{3}$ for the control, unbound RSV, RSV-L and RSV-GL groups, respectively. The improved anticancer efficacy of RSV-GL was attributed to the specific affinity of GE11 towards the overexpressed EGFRs in the cancer cells. The presence of PEG on the outer surface and smaller particle size attributed to the decreased tumor burden (36-38). The histological analysis was performed by H\&E staining analysis (Fig. 7B). The tumors in the control group exhibited darker stained nuclei, indicating continuous tumor cell proliferation, whereas RSV-GL demonstrated high numbers of apoptotic nuclei and a marked decrease in the proportion of cancerous cells.

In conclusion, the GE11-conjugated PEGylated liposome was successfully prepared to enhance the therapeutic effect of RSV in head and neck cancer cells. The EGFR-overexpressing SCC HN cells specifically internalized the GE11 surface conjugated liposome in a manner that was markedly increased 
compared with that of the non-targeted carrier. Consistently, RSV-GL exhibited a significantly increased cytotoxic effect compared with that of non-targeted NPs. Notably, RSV-GL induced significantly increased proportions of early $(\sim 60 \%)$ and late $(\sim 10 \%)$ apoptotic head and neck cancer cells. To the best of our knowledge, the application and development of an EGFR-targeted peptide-conjugated liposome system for RSV delivery has not been studied previously in the treatment of head and neck cancer. Overall, the nanomedicine strategy described in the present study may potentially advance the chemotherapy-based treatment of head and neck cancer, with promising applications in other EGFR-overexpressing tumors.

\section{Acknowledgements}

Not applicable.

\section{Funding}

The present study was supported by grants from the Shenzhen Peking University-The Hong Kong University of Science and Technology Medical Center (grant nos. 2016YFC0104707, SZSM201512026,ZDSYS201504301045406,2015A030313889, JCY J20170413100222613，JCYJ20170412171856582 and 20171228).

\section{Availability of data and materials}

All data generated and analyzed during the present study are included in this article.

\section{Authors' contributions}

TZ, HF, LL, JP and HX performed the experiments, contributed to data analysis and wrote the manuscript. TY, ZZ, YL, YZ, XB, SZ and YS analyzed the data. YC conceptualized the study design, and contributed to data analysis and experimental materials. All authors read and approved the final manuscript.

\section{Ethics approval and consent to participate}

The animal study was approved by the Institutional Animal Ethical Committee of Shenzhen Peking University-Hong Kong University of Science and Technology Medical Center (Shenzhen, China).

\section{Patient consent for publication}

Not applicable.

\section{Competing interests}

The authors declare that they have no competing interests.

\section{References}

1. Kamangar F, Dores GM and Anderson WF: Patterns of cancer incidence, mortality, and prevalence across five continents: Defining priorities to reduce cancer disparities in different geographic regions of the world. J Clin Oncol 24: 2137-2150, 2006 .
2. Leemans CR, Braakhuis BJ and Brakenhoff RH: The molecular biology of head and neck cancer. Nat Rev Cancer 11: 9-22, 2011.

3. Marur S and Forastiere AA: Head and neck cancer: Changing epidemiology, diagnosis, and treatment. Mayo Clin Proc 83: 489-501, 2008.

4. Vermorken JB and Specenier P: Optimal treatment for recurrent/ metastatic head and neck cancer. Ann Oncol 21 (Suppl 7): vii252-vii261, 2010.

5. Kuczynski EA, Sargent DJ, Grothey A and Kerbel RS: Drug rechallenge and treatment beyond progressionimplications for drug resistance. Nat Rev Clin Oncol 10: 571-587, 2013.

6. Holohan C, Van Schaeybroeck S, Longley DB and Johnston PG: Cancer drug resistance: An evolving paradigm. Nat Rev Cancer 13: 714-726, 2013.

7. Yang CS, Landau JM, Huang MT and Newmark HL: Inhibition of carcinogenesis by dietary polyphenolic compounds. Annu Rev Nutr 21: 381-406, 2001.

8. Joe AK, Liu H, Suzui M, Vural ME, Xiao D and Weinstein IB: Resveratrol induces growth inhibition, S-phase arrest, apoptosis and changes in biomarker expression in several human cancer cell lines. Clin Cancer Res 8: 893-903, 2002.

9. Jiang H, Zhang L, Kuo J, Kuo K, Gautam SC, Groc L, Rodriguez AI, Koubi D, Hunter TJ, Corcoran GB, et al: Resveratrol-induced apoptotic death in human U251 glioma cells. Mol Cancer Ther 4: 554-561, 2005.

10. Buhrmann C, Shayan P, Kraehe P, Popper B, Goel A and Shakibaei M: Resveratrol induces chemosensitization to 5-fluorouracil through up-regulation of intercellular junctions, Epithelial-to-mesenchymal transition and apoptosis in colorectal cancer. Biochem Pharmacol 98: 51-68, 2015.

11. Shen M, Wu RX, Zhao L, Li J, Guo HT, Fan R, Cui Y, Wang YM, Yue SQ and Pei JM: Resveratrol attenuates ischemia/reperfusion injury in neonatal cardiomyocytes and its underlying mechanism. PLoS One 7: e51223, 2012.

12. Ku CR, Lee HJ, Kim SK, Lee EY, Lee MK and Lee EJ: Resveratrol prevents streptozotocininduced diabetes by inhibiting the apoptosis of pancreatic $\beta$-cell and the cleavage of poly (ADP-ribose) polymerase. Endocr J 59: 103-109, 2012

13. Walle T, Hsieh F, DeLegge MH, Oatis JE Jr and Walle UK: High absorption but very low bioavailability of oral resveratrol in humans. Drug Metab Dispos 32: 1377-1382, 2004.

14. Ramasamy T, Kim JH, Choi JY, Tran TH, Choi HG, Yong CS and Kim JO: $\mathrm{pH}$ sensitive polyelectrolyte complex micelles for highly effective combination chemotherapy. J Material Chem B 2: 6324, 2014.

15. Hare JI, Lammers T, Ashford MB, Puri S, Storm G and Barry ST: Challenges and strategies in anti-cancer nanomedicine development: An industry perspective. Adv Drug Deliv Rev 108: 25-38, 2017.

16. Sundaramoorthy P, Ramasamy T, Mishra SK, Jeong KY, Yong CS, Kim JO and Kim HM: Engineering of caveolae-specific self-micellizing anticancer lipid nanoparticles to enhance the chemotherapeutic efficacy of oxaliplatin in colorectal cancer cells. Acta Biomater 42: 220-231, 2016.

17. Allen TM and Cullis PR: Liposomal drug delivery systems: From concept to clinical applications. Adv Drug Deliv Rev 65: 36-48, 2013.

18. Kneidl B, Peller M, Winter G, Lindner LH and Hossann M: Thermosensitive liposomal drug delivery systems: State of the art review. Int J Nanomed 9: 4387-4398, 2014.

19. Mohan A, Narayanan S, Balasubramanian G, Sethuraman S and Krishnan UM: Dual drug loaded nanoliposomal chemotherapy: A promising strategy for treatment of head and neck squamous cell carcinoma. Eur J Pharm Biopharm 99: 73-83, 2016.

20. Ramasamy T, Ruttala HB, Gupta B, Poudel BK, Choi HG, Yong CS and Kim JO: Smart chemistry-based nanosized drug delivery systems for systemic applications: A comprehensive review. J Control Release 258: 226-253, 2017.

21. Ramasamy T, Haidar ZS, Tran TH, Choi JY, Choi HG, Yong CS and Kim JO: Layer-by-layer assembly of liposomal nanoparticles with PEGylated polyelectrolytes enhances systemic delivery of multiple anticancer drugs. Acta Biomaterialia 10: 5116-5127, 2014

22. Rosi NL and Mirkin CA: Nanostructures in biodiagnostics. Chem Rev 105: 1547-1562, 2005.

23. Sheng Q and Liu J: The therapeutic potential of targeting the EGFR family in epithelial ovarian cancer. Br J Cancer 104: 1241-1245, 2011.

24. Vidal F, de Araujo WM, Cruz AL, Tanaka MN, Viola JP and Morgado-Díaz JA: Lithium reduces tumorigenic potential in response to EGF signaling in human colorectal cancer cells. Int J Oncol 38: 1365-1373, 2011. 
25. Acharya S, Dilnawaz F and Sahoo SK: Targeted epidermal growth factor receptor nanoparticle bioconjugates for breast cancer therapy. Biomaterials 30: 5737-5750, 2009.

26. Chen L, She X, Wang T, He L, Shigdar S, Duan W and Kong L: Overcoming acquired drug resistance in colorectal cancer cells by targeted delivery of 5-FU with EGF grafted hollow mesoporous silica nanoparticles. Nanoscale 7: 14080-14092, 2015.

27. Kim MW, Jeong HY, Kang SJ, Choi MJ, You YM, Im CS Lee TS, Song IH, Lee CG, Rhee KJ, et al: Cancer-targeted nucleic acid delivery and quantum dot imaging using EGF receptor aptamer-conjugated lipid nanoparticles. Sci Rep 7: 9474, 2017.

28. Li Z, Zhao R, Wu X, Sun Y, Yao M, Li J, Xu Y and Gu J: Identification and characterization of a novel peptide ligand of epidermal growth factor receptor for targeted delivery of therapeutics. FASEB J 19: 1978-1985, 2005.

29. Peer D, Karp JM, Hong S, Farokhzad OC, Margalit R and Langer R: Nanocarriers as an emerging platform for cancer therapy. Nat Nanotechnol 2: 751-760, 2007.

30. Liu D and Auguste DT: Cancer targeted therapeutics: From molecules to drug delivery vehicles. J Control Release 219: 632-643, 2015.

31. Huang WC, Chen SH, Chiang WH, Huang CW, Lo CL, Chern CS and Chiu HC: Tumor microenvironment-responsive nanoparticle delivery of chemotherapy for enhanced selective cellular uptake and transportation within tumor. Biomacromolecules 17: 3883-3892, 2016.

32. Field LD, Nag OK, Sangtani A, Burns KE and Delehanty JB: The role of nanoparticles in the improvement of systemic anticancer drug delivery. Ther Deliv 9: 527-545, 2018.

33. Ruttala HB, Chitrapriya N, Kaliraj K, Ramasamy T, Shin WH, Jeong JH, Kim JR, Ku SK, Choi HG, Yong CS and Kim JO: Facile construction of bioreducible crosslinked polypeptide micelles for enhanced cancer combination therapy. Acta Biomater 63 : 135-149, 2017.
34. Xu Y, Wang S, Chan HF, Liu Y, Li H, He C, Li Z and Chen M: Triphenylphosphonium-modified poly(ethylene glycol)-poly( $\varepsilon$-caprolactone) micelles for mitochondria- targeted gambogic acid delivery. Int J Pharm 522: 21-33, 2017.

35. Sundaramoorthy P, Baskaran R, Mishra SK, Jeong KY, Oh SH, Kyu Yoo B and Kim HM: Novel self-micellizing anticancer lipid nanoparticles induce cell death of colorectal cancer cells. Colloids Surf B Biointerfaces 135: 793-801, 2015.

36. Ma J, Wu H, Li Y, Liu Z, Liu G, Guo Y, Hou Z, Zhao Q, Chen D and Zhu X: Novel core-interlayer-shell DOX/ZnPc Co-loaded MSNs@pH-sensitive CaP@PEGylated liposome for enhanced synergetic chemo-photodynamic therapy. Pharm Res 35: 57, 2018.

37. Gupta B, Ramasamy T, Poudel BK, Pathak S, Regmi S, Choi JY, Son Y, Thapa RK, Jeong JH, Kim JR, et al: Development of bioactive PEGylated nanostructured platforms for sequential delivery of doxorubicin and imatinib to overcome drug resistance in metastatic tumors. ACS Appl Mater Interfaces 9: 9280-9290, 2017.

38. Tyagi P and Subramony JA: Nanotherapeutics in oral and parenteral drug delivery: Key learnings and future outlooks as we think small. J Control Release 272: 159-168, 2018.

This work is licensed under a Creative Commons Attribution-NonCommercial-NoDerivatives 4.0 International (CC BY-NC-ND 4.0) License. 